\title{
The Solar Corona over the Recent Saros
}

\author{
Jay M. Pasachoff \\ Williams College-Hopkins Observatory, Williamstown, Mass. 01267, L'SA; \\ Institute for Advanced Study, Princeton, N.J. 08540, LSA
}

\begin{abstract}
We are now at both the maximum of the solar activity cycle and at the most populated part of the saros. I discuss the solar corona over the recent saros and its changes with the solar activity cycle. We consider the scientific value of eclipse studies and how they relate to other ongoing coronal studies on the sun and other stars.
\end{abstract}

\section{The saros}

Similarly to the way that the beauty of total solar eclipses depends on the coincidence of the angular sizes in the sky of the sun and the moon, the saros depends on the coincidence between 223 synodic months (the period of the phases), which take 6585.32 days, and 19 eclipse years (returns of the sun through one of the nodes where the lunar orbit meets the ecliptic), which take 6585.78 days. The difference is only 0.46 day. In addition, 242 nodical months (also called draconic months, after the mythological Chinese dragon that eats the sun at eclipses), each of which marks the passage of the moon through the node, are only 0.04 day different from the 223 lunar months. Further, 239 anomalistic months (the period of variation of the moon-earth distance) are also within a fraction of a day, so not only the existence of an eclipse but also its general appearance - total or annular, long or short - repeat with this 18 year 11 1/3-day period, the saros (Pasachoff, 1991a). The current saros has a dozen eclipses in it. Thus 22 July 1990 , saw the repeat after a saros of the eclipse of 11 July 1972 . The 1/3-day brought the eclipse from Canada in 1972 to Finland, the Soviet Union, and the U.S. Aleutians, in 1990. 


\section{The value of eclipse observations}

Total solar eclipses still provide the best view astronomers can get of the middle solar corona. The very lowest corona can be seen from coronagraphs usually out to less than a solar radius. The outermost corona has been visible for long periods from spacecraft, such as Solar Maximum Mission. SMM's coronagraph occulted out to 1.75 solar radii, and additional corona was lost because of diffraction rings. Thus the region between 1.1 and 2.0 solar radii is best observable at eclipses.

Further, no spaceborne coronagraph is now aloft. The corona is observable in $\mathrm{x}$-rays on occasion from rockets, but the frequency with which Golub et al. can make $\mathrm{x}$-ray solar images is similar to that of total solar eclipses - a few minutes about once a year. These $\mathrm{x}$-ray images show only the lower corona, and can be mosaiced nicely with white-light eclipse images whenever a rocket flight can be made on the day of an eclipse (which I therefore suggest as desirable).

Other advantages of eclipse observations include the possibility of taking newly developed equipment into the field. Lead times for space missions now approach 10 years and costs are hundreds of millions of dollars. For less than $0.1 \%$ of this cost, one can take an eclipse expedition into the field. Even allowing for cloudy occasions, solar eclipse expeditions are very cost-effective.

Since different eclipses take place at different times in the 11-year solar activity cycle, studying the corona at different activity phases requires a concerted effort over many years. Studies of the solar corona are made all the more important by our new abilities to observe stellar coronas. Important problems like the cause of coronal heating seem best solvable by increased scrutiny of the sun.

\section{Solar eclipses through the most recent saros}

The 1972 eclipse, one saros ago, took place halfway down the cycle. The major e vent in the saros is the long eclipse that took place on 30 June 1973 in Africa and that will repeat on 11 July 1991 . The year 1973 was a time of low sunspot number; the corona had mainly equatorial str eamers. My own experiments at that time observed the ratio of the [Fe XIII] spectral lines at $10747 \AA$ and $10798 \AA$, whose ratio is especially density sensitive.

Weather at the 1974 eclipse in Australia was cloudy for ground-based observers, preventing measurement of the coronal electron temperature by studying the Doppler scattering of Fraunhofer lines, following calculations by Cram. We hope a sensitive CCD will allow us to make a temperature map by this method in 1991. The 1976 eclipse also occurred in Australia under patchy clouds.

For the 1977 eclipse, we brought two inertial-guidance platforms aboard a ship. We made an image in the [Fe XIII] $10747 \AA$ line through a hole in the clouds (Pasachoff et al., 1978), but the skies were not photometric. We were thus not able to make the desired coronal density map. At the 1979 eclipse, seen through hazy skies over the U.S. and Can ada, the corona took second place to the prominences, which heralded the coming solar maximum. 
The 1980 solar-maximum eclipse crossed from Africa to India. We started testing methods of coronal heating via surface Alfvén waves by searching for $1-\mathrm{Hz}$ oscillations in coronal loops, and found signs of excess power in that frequency range in the Fourier transforms (Pasachoff and Landman, 1984). The isophotes showed the round, solar-maximum appearance of the corona. The 1981 corona was still of the solar-maximum type.

The 1983 eclipse was already at a time of declining solar-activity cycle, as the isophotes showed. Though the eclipse was a long one, the sky was not very clear. We again found excess power at about $1 \mathrm{~Hz}$ (Pasachoff and Ladd, 1987). I also collaborated in interpreting Fabry-Perot profiles in terms of the coronal velocity field (Chandrasekhar et al., 1991) and how it varies over the cycle.

The eclipse of 1984 in Papua New Guinea, much shorter and with inferior weather forecasts, was superior scientifically since the sky was exceptionally clear. Our isophotal contours show that we were close to solar minimum. We have used a computer algorithm to bring out coronal streamers to about 5 solar radii, a range usually reached only with spacecraft, illustrating again how important it is to observe all th e eclipses from the ground. Since then, there was only an inaccessible 1985 eclipse and a 1988 eclipse that crossed Sumatra and the Philippines in mostly cloudy skies.

The 22 July 1990 corona was of the solar-maximum type, with round isophotes except for a major coronal hole. We can expect similar morphology for the 11 July 1991 eclipse. We will continue testing magnetohydrodynamic models of coronal heating with an improved version of our coronal-oscillation experiment (Pasachoff, 1991b). On 30 June 1992, an eclipse will start in Uruguay and cross the Atlantic. On 30 November 1994, a better eclipse will cross South America. Solar observers should take the best advantage of them.

Acknowledgments. The expeditions have been supported by NSF grants AST-7922104, CDP-7922926, PRM-8114631, RII-8304403, ATM-9005194, and USE -9050643; NSF Expedition grants to NCAR; and the National Geographic Society.

\section{References}

Chandrasekhar, T., Desai, J.N., Ashok, N.M., Pasachoff, J.M.: 1991, Solar Phys., in press Pasachoff, J.M.: 1991a, Eclipses of the Sun in The Reference Encyclopedia of Astronomy and Astrophysics, in press

Pasachoff, J.M.: 1991b, Proc. Heidelberg Conf. on Mechanisms of Chrom. and Coronal Heating, ed. by P. Ulmschneider, E. Priest, R. Rosner (Springer-Verlag), in press

Pasachoff, J.M., Landman, D.A.: 1984, Solar Phys. 90, 325

Pasachoff, J.M., Ladd, E.F.: 1987, Solar Phys. 109, 365; 110, 412

Pasachoff, J.M., Sandford, II, M.T., Keller, Jr., C. F., Jr.: 1978, Bull. Am. Astron. Soc. 10,431 